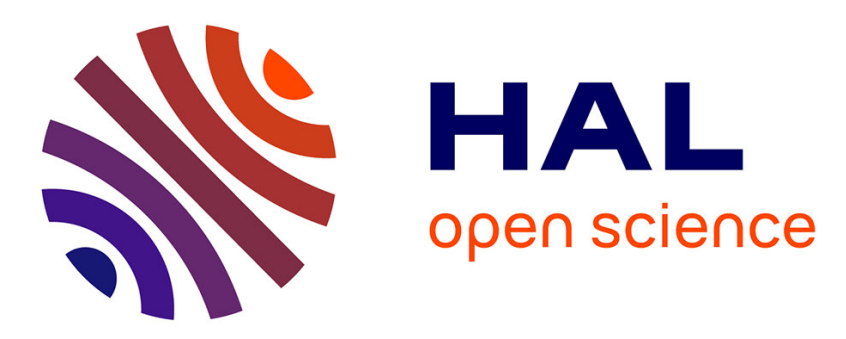

\title{
Analysis of mixing in high-explosive fireballs using small-scale pressurised spheres
}

S. Courtiaud, N. Lecysyn, G. Damamme, Thierry Poinsot, Laurent Selle

\section{To cite this version:}

S. Courtiaud, N. Lecysyn, G. Damamme, Thierry Poinsot, Laurent Selle. Analysis of mixing in high-explosive fireballs using small-scale pressurised spheres. Shock Waves, 2018, pp. 1-15. 10.1007/s00193-018-0814-4 . hal-01743023

\section{HAL Id: hal-01743023 \\ https://hal.science/hal-01743023}

Submitted on 26 Mar 2018

HAL is a multi-disciplinary open access archive for the deposit and dissemination of scientific research documents, whether they are published or not. The documents may come from teaching and research institutions in France or abroad, or from public or private research centers.
L'archive ouverte pluridisciplinaire HAL, est destinée au dépôt et à la diffusion de documents scientifiques de niveau recherche, publiés ou non, émanant des établissements d'enseignement et de recherche français ou étrangers, des laboratoires publics ou privés. 


\section{Open Archive TOULOUSE Archive Ouverte (OATAO)}

OATAO is an open access repository that collects the work of Toulouse researchers and makes it freely available over the web where possible.

This is an author-deposited version published in : http://oatao.univ-toulouse.fr/ Eprints ID : 19758

To link to this article : DOI : 10.1007/s00193-018-0814-4

URL : http://dx.doi.org/10.1007/s00193-018-0814-4

To cite this version : Courtiaud, S. and Lecysyn, N. and Damamme, G. and Poinsot, Thierry and Selle, Laurent Analysis of mixing in high-explosive fireballs using small-scale pressurised spheres. (2018) Shock Waves. pp. 115. ISSN 0938-1287

Any correspondence concerning this service should be sent to the repository administrator: staff-oatao@ listes-diff.inp-toulouse.fr 


\title{
Analysis of mixing in high-explosive fireballs using small-scale pressurised spheres
}

\author{
S. Courtiaud ${ }^{1} \cdot$ N. Lecysyn ${ }^{1}$ (D) G. Damamme ${ }^{1} \cdot$ T. Poinsot $^{2}\left(\right.$ D $\cdot$ L. Selle ${ }^{2}(D)$
}

Received: 25 September 2017 / Revised: 22 January 2018 / Accepted: 29 January 2018

(c) Springer-Verlag GmbH Germany, part of Springer Nature 2018

\begin{abstract}
After the detonation of an oxygen-deficient homogeneous high explosive, a phase of turbulent combustion, called afterburning, takes place at the interface between the rich detonation products and air. Its modelling is instrumental for the accurate prediction of the performance of these explosives. Because of the high temperature of detonation products, the chemical reactions are mixing-driven. Modelling afterburning thus relies on the precise description of the mixing process inside fireballs. This work presents a joint numerical and experimental study of a non-reacting reduced-scale set-up, which uses the compressed balloon analogy and does not involve the detonation of a high explosive. The set-up produces a flow similar to the one caused by a spherical detonation and allows focusing on the mixing process. The numerical work is composed of 2D and 3D LES simulations of the set-up. It is shown that grid independence can be reached by imposing perturbations at the edge of the fireball. The results compare well with the existing literature and give new insights on the mixing process inside fireballs. In particular, they highlight the fact that the mixing layer development follows an energetic scaling law but remains sensitive to the density ratio between the detonation products and air.
\end{abstract}

Keywords Homogeneous high explosives · Afterburning · Turbulent mixing · LES · Compressed balloon method

\section{Introduction}

Despite a continuous effort, the calculation of the blast produced by an oxygen-deficient high explosive (HE) is still a challenge. Contrary to ideal HEs for which all the chemical energy is liberated during the detonation, oxygen-deficient HEs generate detonation products that can still exothermically react with air during a phase called afterburning, which results in an enhancement of the blast. Detonation products appear as an intensely glowing, burning cloud of gas and soot called a fireball.

Previous studies have shown that the combustion process inside fireballs is mainly driven by the turbulent mixing

Communicated by M. Brouillette and A. Higgins.

S. Courtiaud

sebastien.courtiaud@cea.fr

L. Selle

laurent.selle@imft.fr

CEA, DAM, CEA-Gramat, 46500 Gramat, France

2 IMFT, UMR CNRS/INP-UPS 5502, Allée du professeur Camille Soula, 31400 Toulouse, France of detonation products with air [1]. Rayleigh-Taylor (RT) instabilities [2,3] and, then, Richtmyer-Meshkov (RM) instabilities $[4,5]$ allow the growth of a turbulent mixing layer at their edge. The mixing process is thus a key parameter in the phenomenology of fireballs as it controls both the flame mean radius, its wrinkling, and the local speed of sound. Consequently, it also drives the total heat released by afterburning reactions and the blast profile.

Very recent 3D numerical simulations of TNT explosions performed by Milne et al. [6] seem to confirm the key role of turbulent mixing. They indeed obtained results in good agreement with experimental data by resolving the mixing layer while considering instantaneous chemical reactions.

The objective of the present study is to better understand the mixing processes occurring inside HE fireballs by using a non-reacting reduced-scale set-up. This study relies on both numerical work and experimental work. The set-up, which is essentially a spherical shock tube similar to the one used by Boyer [7] in 1959, consists of bursting air-pressurised glass spheres. Even though it does not involve the detonation of a HE, it produces a similar flow, which is representative of the one following a spherical detonation [8]. This analogy is often referred to as the compressed balloon method. The 
numerical study consists in two-dimensional (2D) and threedimensional (3D) large-eddy simulations (LES) of the set-up and allows investigating the influence of the initial density ratio and energy, which are key parameters in the development of the mixing layer.

The results of the numerical and experimental work are presented and compared in this paper. In Sect. 2, the phenomenology associated with afterburning is described in detail. In Sect. 3, the configuration, experimental set-up and numerical methods are described. Then, the results of the 2D numerical study, which focuses on mesh independence, are presented and discussed in Sect. 4. Section 5 is dedicated to the analysis of the experimental data, and finally, in Sect. 6, the results of the 3D numerical study are presented and compared to the experiment. Throughout this study, specific attention is paid to the effect of the initial energy and density ratio.

\section{Phenomenology}

\subsection{Flow induced by a spherical detonation}

After the spherical detonation of a HE, the expansion of detonation products creates a complex flow for which four characteristic steps have been identified by Anisimov et al. [9,10] and Kuhl et al. [11,12] (Fig. 1). First, during what is called the strong blast wave phase, an intense shock wave is transmitted into the atmosphere, and the interface between the detonation products and air is swept outwards. This region is referred to as the interface or the mixing layer in the remainder of the text, and the objective of this work is to understand its dynamics. Perturbations of the interface due, for example, to local inhomogeneities in the explosive material $[11,13]$ arise, but their amplitude remains small. An expansion wave propagates inwards. Simultaneously, due to the spherical expansion of the detonation products, the pressure gradient close to the interface builds up which creates a secondary shock wave inside the fireball. After the expansion wave has reflected at the centre of the fireball, it starts propagating outwards and interacts successively with the secondary shock wave and the interface. The flow is reversed behind the expansion wave so that the radii of the secondary shock wave and the mixing region then start to decrease. This phase is referred to as the implosion phase. The deceleration of the interface allows the growth of flow structures in the mixing layer from the small initial perturbations through RT instabilities [2,3] since the interface is naturally unstable [14]. The combustion of detonation products in a turbulent diffusion flame brings energy to the mean flow, which results in a lower pressure drop behind the primary shock wave. The inward-propagating secondary shock wave is then reflected at the centre and starts propagating outwards. Its interac-

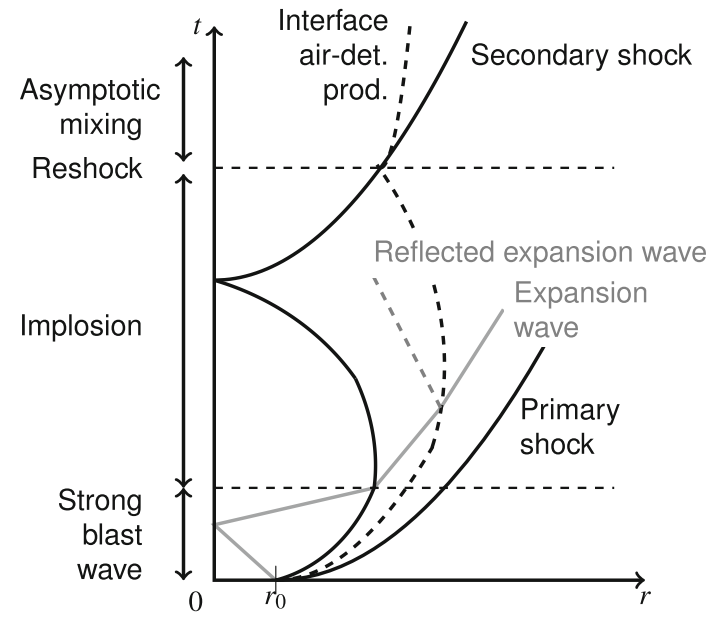

Fig. 1 Theoretical time-versus-distance wave diagram of a cylindrical or spherical explosion

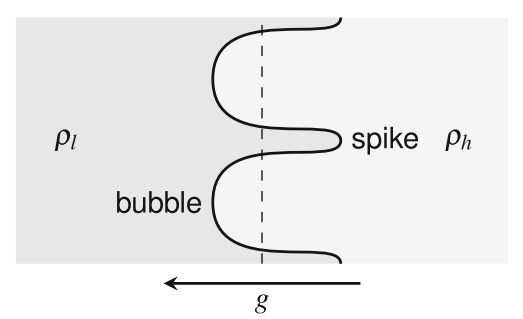

Fig. 2 Schematic representation of the structures resulting from the RT instability

tion with the mixing layer, called the reshock phase, causes the fireball to grow again. Mixing is enhanced due to RM instabilities [4,5] resulting in a further increase of the reaction rate. Eventually, an asymptotic mixing phase is reached when the radius of the fireball becomes steady, even though combustion still occurs. The heat released by afterburning yields an improved blast and an augmented impulse, which is defined as the temporal integral of the positive phase of the overpressure signal (i.e., $P-P_{0}>0$ ).

\subsection{Rayleigh-Taylor instability}

Mixing in HE fireballs is mainly driven by the RT instabilities occurring during the strong blast wave phase and the implosion phase. RT instability is caused by the acceleration of a heavy fluid (density $\rho_{\mathrm{h}}$ ) by a lighter one (density $\rho_{\mathrm{l}}$ ). It develops at the interface between the two fluids from small initial perturbations, which cause a misalignment of the density and pressure gradients. It is present without heat release. The associated baroclinic torque gives birth to vortical structures resulting in the formation of bubbles of light fluid into the heavy one, and spikes of heavy fluid into the light one, as represented in Fig. 2. 
Three characteristic steps of development of the RT instability have been identified by Youngs [15] for small initial perturbations. He stated that the effects of viscosity result in the appearance of a most unstable mode of wavelength $\lambda_{\mathrm{m}}$, which corresponds to the initially fastest growing perturbations. For a planar interface submitted to a constant acceleration $g$, one has:

$\lambda_{\mathrm{m}} \approx 4 \pi\left(\frac{v^{2}}{g A}\right)^{\frac{1}{3}}$,

where $A=\left(\rho_{\mathrm{h}}-\rho_{\mathrm{l}}\right) /\left(\rho_{\mathrm{h}}+\rho_{1}\right)$ is the Atwood number, and $v$ is the average kinematic viscosity defined as:

$v=\frac{\mu_{\mathrm{h}}+\mu_{1}}{\rho_{\mathrm{h}}+\rho_{\mathrm{l}}}$,

in which $\mu_{\mathrm{h}}$ and $\mu_{1}$ are the respective dynamic viscosity of the heavy fluid and the light fluid.

First, perturbations corresponding to the most unstable wavelength grow symmetrically in shape and exponentially in time until their amplitude $h$ is such that $h \approx 1 / 2 \lambda_{\mathrm{m}}$. In a second phase, nonlinear effects cause the saturation of their growth and their shape becomes asymmetric. If the range of wavelengths in the initial perturbation is large, and since large structures develop more slowly than small ones during the initial exponential growth, but faster after saturation, the flow is then dominated by larger and larger structures until the wavelength of the dominant structure $\lambda_{d}$ is approximately $10 \lambda_{\mathrm{m}}$. From this point, the knowledge of the initial state is lost and the evolution of the instability is self-similar, i.e., the bubbles height and diameter are proportional. Under a constant acceleration $g$, the height $h_{\mathrm{b}}$ (respectively, $h_{\mathrm{s}}$ ) of the bubbles (respectively, spikes) evolves quadratically with time [15-17]:

$h_{\mathrm{b}, \mathrm{s}} \approx \alpha_{\mathrm{b}, \mathrm{s}} A g t^{2}$

where $\alpha_{\mathrm{b}}$ and $\alpha_{\mathrm{s}}$ are the growth rates of bubbles and spikes, respectively.

In the case of a cylindrical or spherical geometry, the initial growth of the instability from small perturbations is described by the Bell-Plesset equation [14,18] and is no longer exponential but follows instead a Gauss hypergeometric function $[19,20]$. However, this equation tends towards the one for the planar case when the wavelength of the perturbations is small compared to the radius of curvature of the interface.

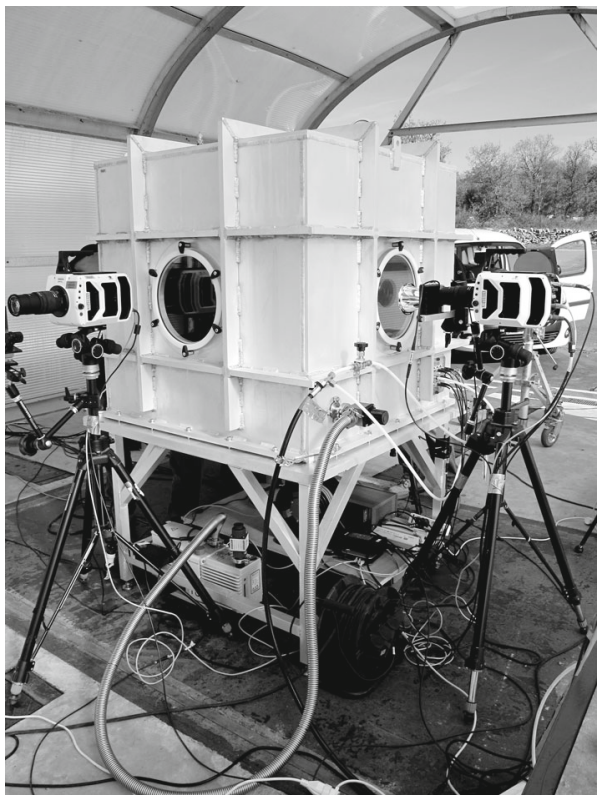

Fig. 3 View of the exterior of the vessel showing the two high-speed cameras used for schlieren (left) and glass fragments observation (right)

\section{Methodologies and configurations}

\subsection{Experimental set-up}

The experimental study of HE afterburning faces multiple technical challenges. First the timescales are very small, requiring data acquisition rates as large as $1 \mathrm{GHz}$. Then, the large power density, the presence of shock waves, and the projection of debris call for specific protection measures for the diagnostics. Finally, the opacity of the fireball due to soot hinders the observation of mixing processes. Therefore, this study aims at reproducing similar flows, at a reduced scale, by bursting air-pressurised glass spheres. The experimental data are used as a reference for a numerical study and allows testing the influence of the initial density ratio and energy on the mixing process.

The experimental set-up consists of two main components: a glass sphere pressurised with air and a steel vessel with optical access, shown in Fig. 3, for the protection of the optical diagnostics. The spheres are made of hand-blown $\approx 1$-mm-thick borosilicate glass, sealed to a metallic base equipped with a gas inlet. They are mounted on the top of a mast at the centre of the vessel. The vessel is cubic and $1 \mathrm{~m}$ wide. Three faces are equipped with windows to allow optical access. An optical head used for photonic Doppler velocimetry (PDV) is mounted on the upper face. A pneumatic hammer, mounted on the mast, was designed to break the sphere once it is pressurised. However, as the spheres were too fragile to sustain the desired level of pressure, it has never been used in practice (see Sect. 5). 


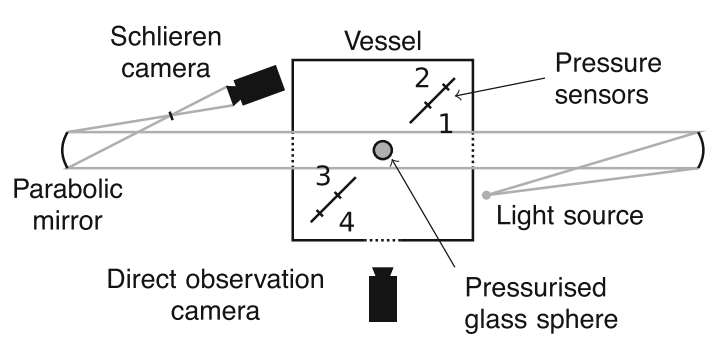

Fig. 4 Experimental set-up as seen from above

A schematic of the installation is represented in Fig. 4. The diagnostics are: (1) a high-speed numerical schlieren camera to study the flow and determine the wave diagram, (2) pressure sensors to measure the blast, and (3) a high-speed video of the sphere and PDV to determine the velocity of the glass fragments.

Both videos are recorded at a rate of 75,000 frames per second and a resolution of $512 \times 512$ pixels, which corresponds to around $0.4 \mathrm{~mm}$ per pixel. Four pressure sensors are used and placed on two pencil gauges. Two (numbers 1 and 3 in Fig. 4) are located at $0.362 \mathrm{~m}$ from the centre of the sphere, and two (numbers 2 and 4 ) at $0.422 \mathrm{~m}$. A copper tape is stuck to the sphere at the impact location of the hammer. Both tape and hammer are part of the triggering circuit so that measurements are triggered when the hammer and the tape come into contact and close the circuit.

The experimental wave diagram is obtained by measuring the shocks and interface positions on the high-speed camera frames after having calibrated them to determine their effective resolution. The reference length used for calibration is the sphere diameter.

\subsection{Numerical method}

The numerical study is conducted with the AVBP solver [21] in the framework of large-eddy simulation (LES). The compressible Navier-Stokes equations are solved using a two-step Taylor-Galerkin low-dissipation centred scheme called TTG4A [22,23], which is third order in space and fourth order in time. No subgrid scale model is used for the 2D computations, and the sigma subgrid scale model [24] is used for the 3D simulations. This model has the advantage of introducing no subgrid scale viscosity for pure diverging and rotating flows, which is well adapted to the configurations studied in this paper. The gas is represented using a multispecies approach and a perfect gas equation of state with temperature-dependent heat capacities and transport coefficients.

The meshes are unstructured and divided into two zones: a spherical one, well resolved, centred on the sphere, and wide enough to cover the area swept by the mixing layer, and a second one ensuring a continuous transition to the boundaries of the domain, where the cells are larger. For the 2D simulations, the domain is a disc of diameter $90 \mathrm{~cm}$, and a free outlet boundary condition is imposed on the boundary of the domain. For the 3D simulations, the domain is a cube (side of length $0.5 \mathrm{~m}$ ) representing one-eighth of the experimental confinement vessel. An adiabatic slip wall boundary condition is imposed on the three faces representing the walls of the vessel, and the three other faces are treated as symmetries.

In order to reproduce the experimental initial conditions, the initial temperature is uniform in the computational domain $(299 \mathrm{~K})$ and the velocity is zero everywhere. The initial pressure in the vessel is set to $1.0 \mathrm{bar}$, and a constant pressure $P_{1}$ is imposed inside a spherical zone representing the glass sphere. The resulting pressure jump is smoothed over a few cells via a hyperbolic tangent function in order to reduce spurious numerical oscillations at the start-up of the computation. For the 2D simulations, the high-pressure zone is located at the centre of the domain, whereas, for the 3D ones, it is located in the corner representing the centre of the vessel. The glass from which the spheres are made and the mast are not taken into account in the simulations.

The numerical wave diagram is obtained by post-processing either the whole field in the case of $2 \mathrm{D}$ simulations or $2 \mathrm{D}$ cuts of the $3 \mathrm{D}$ field. First, an azimuthal average, $\bar{f}$, and root mean square, $f_{\text {rms }}$, of the flow variables $f=$ $\rho, P, T, u_{r}, u_{\theta}, u_{\varphi}, Y_{k}$ are computed on spherical shells. $\rho$ is the density of the fluid, $P$ is the pressure, and $T$ is the temperature. $u_{r}, u_{\theta}, u_{\varphi}$ designate, respectively, the radial, the azimuthal, and the polar components of the flow velocity, and $Y_{k}$ is the mass fraction of species $k$. Then, the shocks and interface positions are determined by using dedicated numerical sensors. The shock sensor is similar to the one proposed by von Neumann and Richtmyer [25] for shock handling in numerical simulations. It allows the determination of the shock position by peak detection. It makes use of the gradient of the mean radial velocity $\overline{u_{\mathrm{r}}}$ and is positive only in compression regions. Its expression is:

$\eta_{\text {shock }}=\frac{\partial \overline{u_{r}}}{\partial r}\left(\frac{\partial \overline{u_{r}}}{\partial r}-\left|\frac{\partial \overline{u_{r}}}{\partial r}\right|\right)$

The gas composition inside and outside the glass sphere is initially identical. However, in the numerical simulation, two oxygen species (with identical thermodynamic and transport properties) have been artificially defined, thus allowing the quantitative measurement of their mixing. The interface corresponds to the region where these two species mix, and is defined as the transition zone where $0.1 Y_{\mathrm{O}_{2}, 0} \leqslant \bar{Y}_{\mathrm{O}_{2}} \leqslant$ $0.9 Y_{\mathrm{O}_{2}, 0}$, with $Y_{\mathrm{O}_{2}, 0}$ the initial mass fraction of oxygen in the vessel. Changing the threshold values changes the quantitative evaluation of the thickness of the mixing layer (by a few percent) but not the relative evolution between cases. 
Table 1 Studied configurations

\begin{tabular}{llll}
\hline Case & $P_{1}($ bar $)$ & $\varnothing(\mathrm{mm})$ & $A$ \\
\hline A & 22.5 & 51 & 0.91 \\
B & 22.5 & 73 & 0.91 \\
C & 45.0 & 51 & 0.96 \\
\hline
\end{tabular}

\subsection{Configurations}

For a given HE, considering an unconfined spherical explosion, the only parameter impacting the afterburning process is the mass of the charge. However, a change in mass causes both a change in the total energy and a change in the density ratio at the interface between detonation products and air. Indeed, the density of the detonation products is a function of the detonation velocity, which depends on the charge radius. Using the compressed balloon analogy, it is possible to decouple these two parameters, which are also the ones driving the growth of RT instabilities, as recalled in Sect. 2. Therefore, three configurations, listed in Table 1, have been studied and simulated numerically. They allow the independent investigation of the influence of the initial energy (through a variation of the initial inner sphere diameter, $\varnothing$, case B) and the initial density of the pressurised gas on the mixing process (case $\mathrm{C}$ ). Case $\mathrm{A}$ is the reference one, mimicking the conditions of the study of Boyer [7] for crossvalidation.

\subsection{Scaling law}

In order to compare the experimental and numerical results, and the different configurations in the same frame, Sachs scaling law [26] is used and adapted to pressurised vessels. Scaling laws are derived from the conservation of momentum so that, for a given type of energy source (here pressurised glass spheres), the blast profile in scaled coordinates only depends on the scaled distance and is independent of the initial energy. Time and distance are rescaled according to the following equations [27]:

$$
\begin{aligned}
& r \rightarrow \lambda=\left(2 \pi(j-1) \frac{P_{0}}{E_{1, j}}\right)^{\frac{1}{j}} r=s_{r} r \\
& t \rightarrow \tau=c_{0}\left(2 \pi(j-1) \frac{P_{0}}{E_{1, j}}\right)^{\frac{1}{j}} t=s_{t} t
\end{aligned}
$$

where $j=2$ for 2D axisymmetrical flows and $j=3$ for 3D spherically symmetrical flows, and where
Table 2 Scaling coefficients

\begin{tabular}{llllll}
\hline Case & $2 \mathrm{D}$ & & $3 \mathrm{D}$ & \\
\cline { 2 - 3 } \cline { 5 - 6 } & $s_{r}\left(\mathrm{~m}^{-1}\right)$ & $s_{t}\left(\mathrm{~s}^{-1}\right)$ & & $s_{r}\left(\mathrm{~m}^{-1}\right)$ & $s_{t}\left(\mathrm{~s}^{-1}\right)$ \\
\hline A & 7.4 & 2563.5 & 14.8 & 5117.3 \\
B & 5.2 & 1790.9 & 10.3 & 3575.1 \\
C & 5.2 & 1812.7 & 11.7 & 4061.6 \\
\hline
\end{tabular}

$E_{1, j}(j=2)=\pi r_{1}^{2} \frac{P_{1}}{\gamma_{1}-1}$

$E_{1, j}(j=3)=\frac{4}{3} \pi r_{1}^{3} \frac{P_{1}}{\gamma_{1}-1}$

is the total energy of the gas initially contained in the sphere. $c_{0}$ is the sound speed, and $r_{1}$ is the initial sphere radius. The subscripts 0 and 1 refer to the fluid outside and inside the sphere, respectively. The scaling coefficients used for cases $\mathrm{A}, \mathrm{B}$, and $\mathrm{C}$ for both the $2 \mathrm{D}$ and $3 \mathrm{D}$ geometries are given in Table 2 .

\section{2D numerical study}

The objective of the 2D study is to investigate the effects of the mesh resolution on the mixing process and the influence of initial geometric perturbations of the interface on the development of RT instabilities in the mixing layer.

In the following, for clarity, computations will be referenced using a notation of the form $\mathrm{C}_{-} \mathrm{M} x_{-} \mathrm{A} y . \mathrm{C}$ is the label of the case, $\mathrm{M}$ refers to the cells size in the central area of the mesh, and A refers to the initial amplitude of the geometric perturbations imposed on the interface. The values $x$ and $y$ associated with the last two parameters are both given in tenths of a millimetre. For example, the computation referenced as A_M5_A10 is a simulation of case A performed on the grid with a cell size $\Delta=0.5 \mathrm{~mm}$ and an initial amplitude of perturbations $h_{0}=1.0 \mathrm{~mm}$.

\subsection{Global phenomenology}

Without geometric perturbation, i.e., when the interface is initially round, the natural instability of the interface is captured by the simulations. Figure 5 presents, in logarithmic scale, the density gradient obtained with simulation A_M2.5_A0.

For this simulation without initial perturbations, the instability grows from the numerical roundoff errors. Since the interface is naturally unstable, as predicted by the theory on RT instabilities [14,19], these small errors are sufficient to give birth to a highly convoluted, seemingly turbulent mixing layer. Figure 5 also highlights the evolution of the primary and secondary shock waves as well as their interaction with the mixing layer. The secondary shock wave is moving out- 


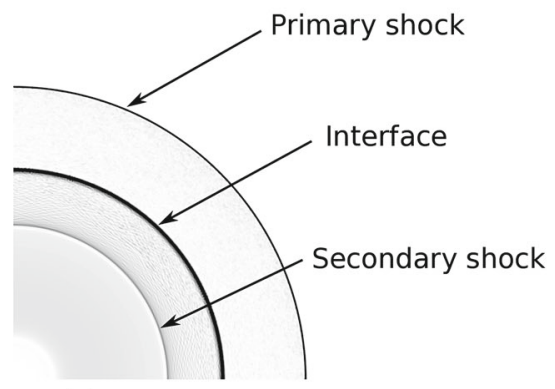

(a) Strong blast wave phase

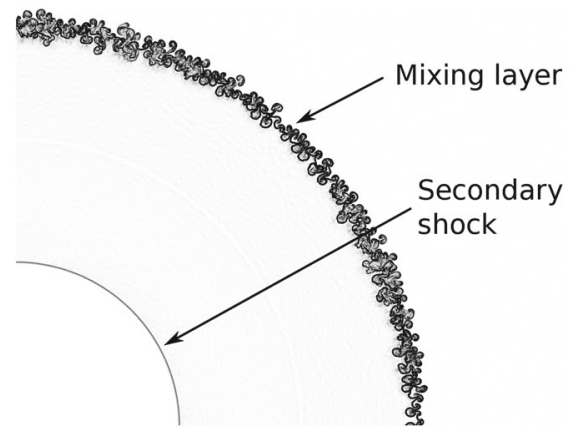

(b) Beginning of the implosion phase

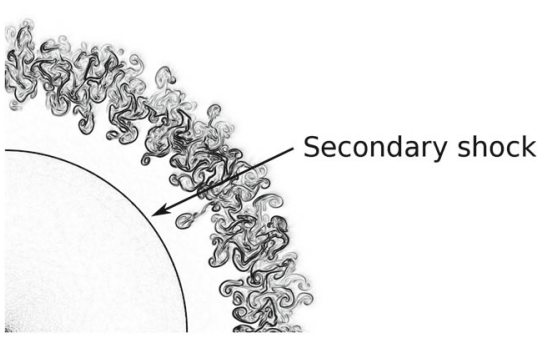

(c) End of the implosion phase

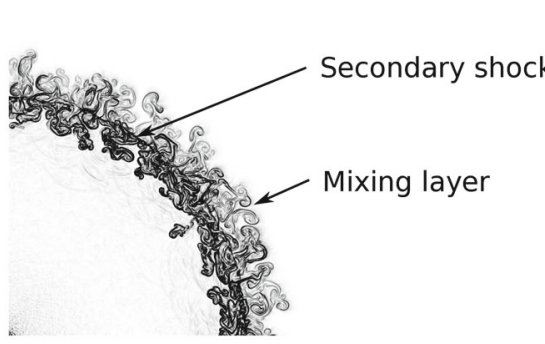

(d) Reshock phase

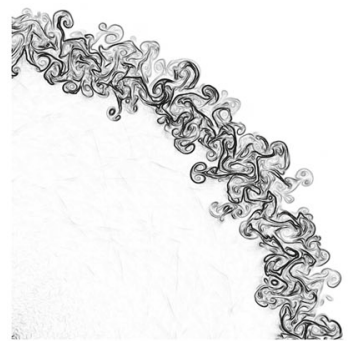

(e) Asymptotic mixing phase

Fig. 5 Density gradient in logarithmic scale obtained with 2D simulation A_M2.5_A0 showing the characteristic steps of the flow. Zoom on one quarter (the computational domain includes the full disc). All pictures have the same scale

wards in Fig. 5a, inwards in Fig. 5b and outwards again in Fig. 5c, d.

The four characteristic phases presented in Sect. 1 (strong blast wave, implosion, reshock, and asymptotic mixing) can also clearly be identified on the wave diagram presented in Fig. 6a, which is obtained using data from calculation A_M2.5_A10, i.e., with initial perturbations. The implosion phase begins at $\tau=1.0$ when the expansion wave (not tracked) interacts with the mixing layer internal border, which translates into a decrease of its radius. The reshock phase begins when the secondary shock wave enters the mixing layer at $\tau=2.3$ and ends when it emerges from the mixing layer at $\tau=2.7$. The evolution of the mixing layer thickness $\eta=s_{r}\left(r_{\text {int, } m a x}-r_{\text {int, min }}\right)$, plotted in Fig. 6b, is first exponential during the strong blast wave phase and is quite linear during the implosion and reshock phases. As the acceleration of the interface is quite constant during the strong blast wave phase, and since the wavelength of the perturbations is small compared to the interface radius $\left(\lambda_{\max }<0.1 r_{1}\right.$, see Sect. 4.3), the initial evolution of the mixing layer thickness is consistent with the theory on RT instability.

Figure $6 \mathrm{c}$ shows the temporal variation of the turbulent kinetic energy in the computational domain. One can see a fast exponential growth during the strong shock phase, which turns into a fairly linear increase during the implosion phase, and then a stagnation during the last two phases. About half of the turbulent kinetic energy is produced during the strong blast wave phase and the second half is produced during the implosion phase, i.e., while the interface is strongly decelerated. The initial exponential increase is, for example, consistent with simulations of HE fireballs performed by Kuhl et al. [11,28].

Figure $6 \mathrm{~d}$ shows the evolution of the ratio between the actual area of the mixing layer internal boundary (i.e., the isosurface $\left.Y_{\mathrm{O}_{2}}=0.1 Y_{\mathrm{O}_{2}, 0}\right)$ and the area of the sphere whose radius is equal to the internal boundary mean radius. This parameter gives an indication of the wrinkling of the internal boundary and is useful to understand the afterburning phenomenon. The flame in homogeneous HE fireballs is indeed of the diffusion type: it is located in the mixing layer, on the stoichiometric isosurface, whose shape is governed by the local flow. As the stoichiometric zone is suspected to be located close to the internal boundary of the mixing layer, its wrinkling indicates how the flame would behave in a fireball. The surface ratio appears to grow quadratically during the strong blast wave phase and linearly during the implosion phase. It then decreases almost linearly during the reshock phase and is almost constant afterwards.

\subsection{Mesh influence without initial perturbation}

In order to evaluate the influence of mesh resolution on the simulation of the mixing layer, computations of case A were performed on several meshes having the same topology but 


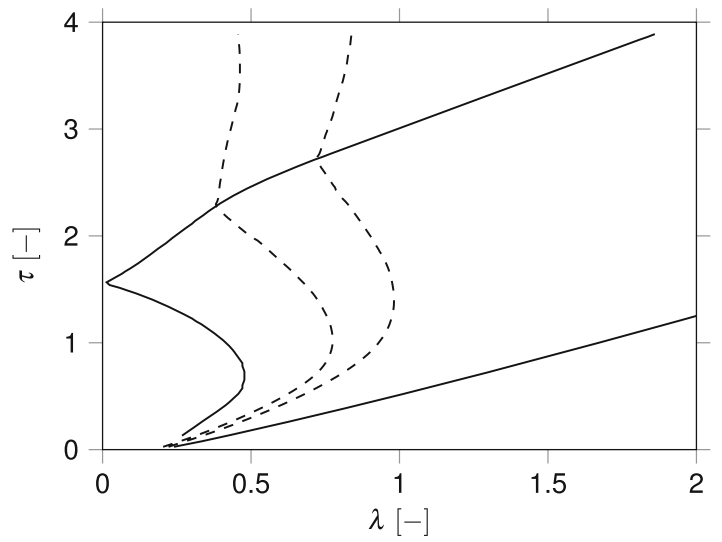

(a) Wave diagram. - : shocks,

-- - : mixing layer boundaries.

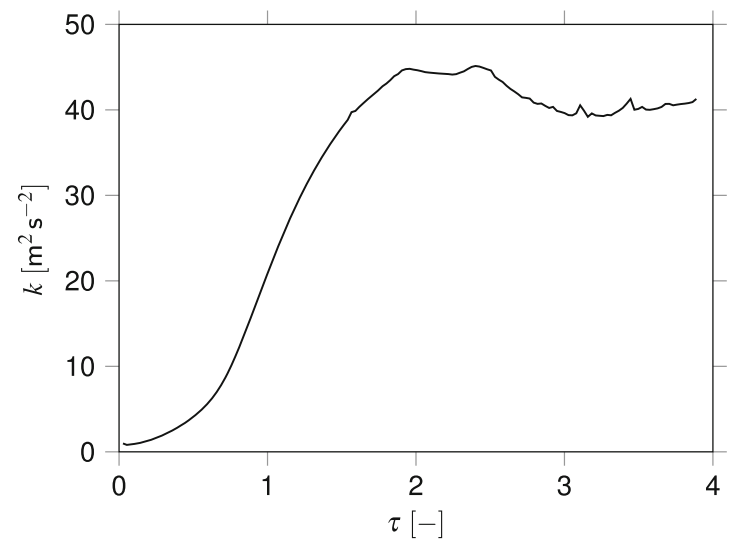

(c) Turbulent kinetic energy

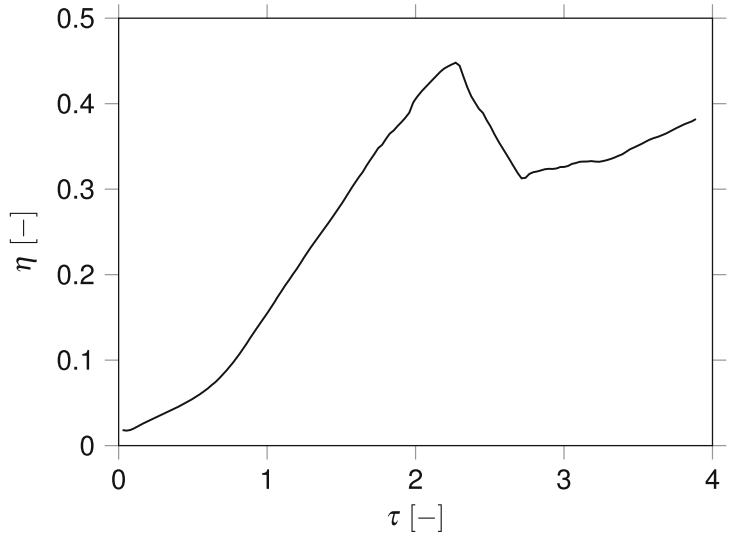

(b) Mixing layer thickness

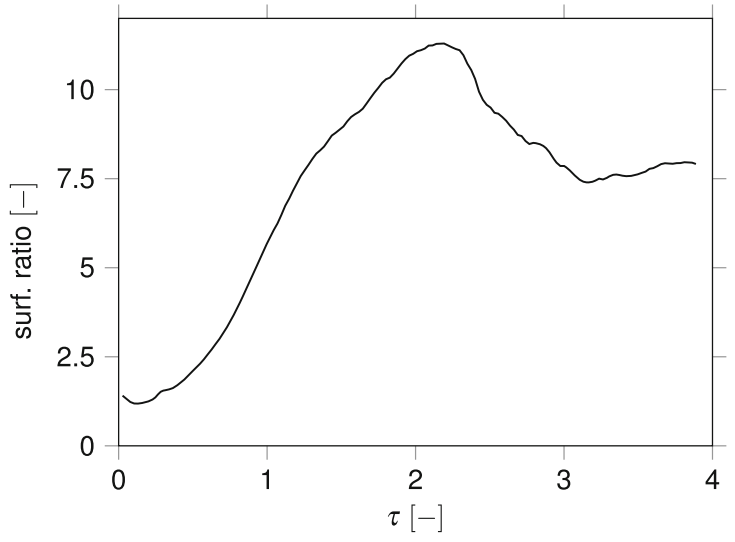

(d) Surface ratio

Fig. 6 Temporal evolution of main characteristics of simulation A_M2.5_A10

a different resolution in the central area. Resolutions of 0.5 , 0.25 , and $0.125 \mathrm{~mm}$ were used corresponding to meshes with $1,2.5$, and 8 million cells, respectively. For these simulations, no perturbation is imposed so that the interface is initially circular. The effect of spatial resolution is assessed by comparing the wave diagrams, the evolution of the mixing layer thickness $\eta$ and the evolution of the turbulent kinetic energy, $k$.

The wave diagrams, presented in Fig. 7, show that the mesh resolution has no visible influence on the shocks but mildly thickens the mixing layer, especially its external boundary. The evolution of the mixing layer thickness shown in Fig. 8 illustrates that a larger cell size increases the growth rate during the implosion phase. This phenomenon is caused by the difference in the wavelength of numerical perturbations resolved on each grid. The wavelength of the most unstable mode $\lambda_{\mathrm{m}}$ can be estimated with (1), which is however established for the planar case since an equivalent expression for the cylindrical geometry is not available. Considering the initial acceleration $g$, which is given by [10]: $g=\frac{c_{1}^{2}}{r_{1}}\left(\frac{\rho_{0}}{\rho_{1}}\right)^{\frac{1}{2}}$

where $c_{1}$ is the sound speed inside the sphere, the most unstable wavelength for case $\mathrm{A}$ is found to be $\lambda_{\mathrm{m}}=1.56 \times$ $10^{-2} \mathrm{~mm}$, which is not resolved on any of the present grids. It should however be noticed that this wavelength is several orders of magnitude lower than the characteristic size of the debris that are observed experimentally $(\sim 10 \mathrm{~mm})$. The effective most unstable mode is then the one with the lowest wavelength that can be resolved on the grid, which is, for a mesh with cells size $\Delta$, the one corresponding to $\lambda=4 \Delta$. Since the most unstable mode has a lower wavelength on a more refined grid, it grows faster, but it also saturates faster and for a lower amplitude (i.e., when $h \approx 0.5 \lambda$ ). After saturation, a higher wavelength leads to a faster growth of the turbulent structures. Therefore, the mixing layer thickness grows faster on a coarser mesh. Since saturation occurs before the beginning of the implosion phase, it explains why the influence of the resolution is most visible during that phase. Accordingly, the turbulent kinetic energy, shown in 


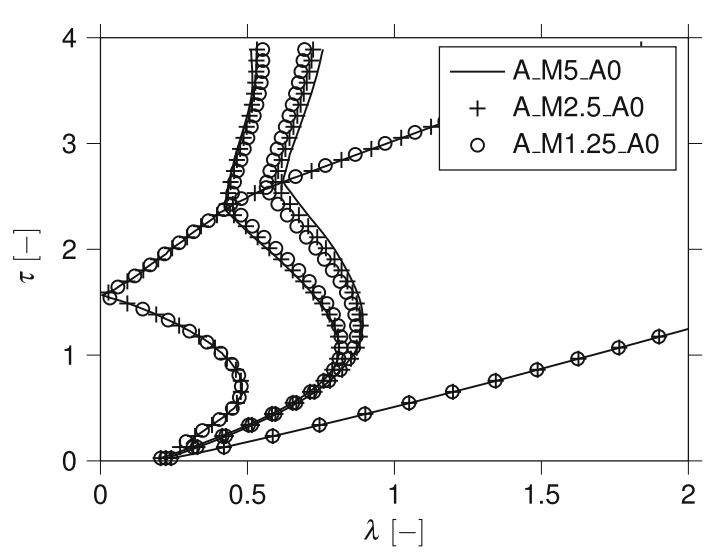

Fig. 7 Influence of the mesh resolution on the wave diagram without initial geometric perturbations ( $2 \mathrm{D}$ case)

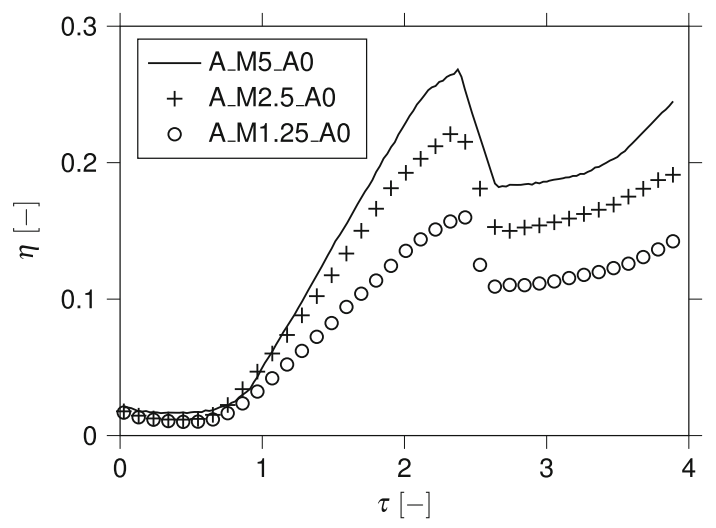

Fig. 8 Influence of the mesh resolution on the mixing layer thickness evolution without initial geometric perturbations (2D case)

Fig. 9, grows faster and reaches higher levels on the coarsest mesh. This preliminary study of the influence of spatial resolution in the $2 \mathrm{D}$ case leads to the conclusion that mesh convergence for a case without initial perturbations is far from being reached. A 3D study might be in order, but first it is out of reach in terms of computational power and, more importantly, it will now be shown that adding perturbations to the initial field has a first-order influence on the evolution of the mixing process.

\subsection{Effect of initial geometric perturbations}

Using the same three meshes, the initial location of the interface is perturbed by a sum of sinusoids:

$r_{\mathrm{int}, i}=r_{\mathrm{int}, 0}+\frac{h_{0}}{2} \frac{\sum_{n} \cos \left(n \theta+\phi_{n}\right)}{\max \left(\sum_{n} \cos \left(n \theta+\phi_{n}\right)\right)}$

where $\theta$ is the azimuth, $r_{\text {int, } 0}$ is the interface radius without initial perturbations, $h_{0}$ is the initial amplitude, chosen to be identical for all modes, $n$ is an integer designating the mode number, and $\phi_{n}$ is a phase associated with mode $n$. The phase

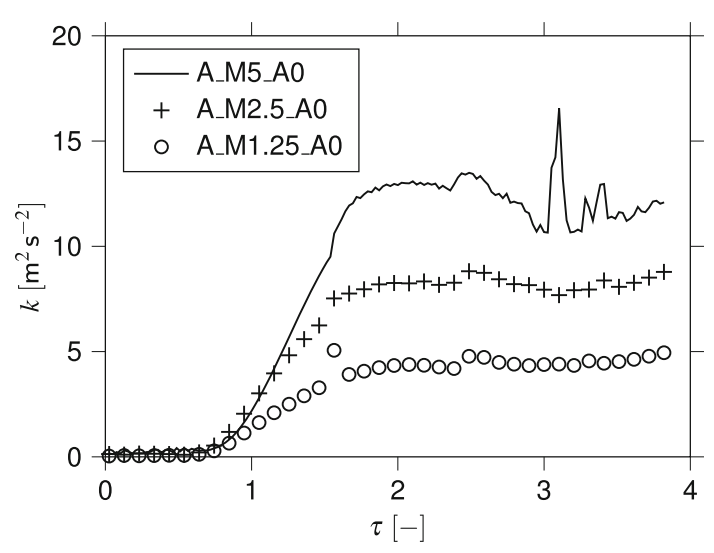

Fig. 9 Influence of the mesh resolution on the turbulent kinetic energy evolution without initial geometric perturbations (2D case)

is chosen randomly for each mode, but the set of phases is the same for all the simulations.

Two constraints on the perturbation wavelengths are to be considered. First, they have to be small enough so that the structures they create can interact to form a turbulent mixing layer in a self-similar regime instead of a succession of independent mushroom-like structures. Then, the wavelengths must be large enough to be resolved on the grid. This last constraint also applies to the amplitude of the perturbations. Consequently, $n$ is chosen between 40 and 80 which corresponds, for the sphere diameter considered in case $\mathrm{A}$, to wavelengths varying from 4 to $2 \mathrm{~mm}$, which is the minimal wavelength that can be resolved on the coarsest grid. The initial amplitude $h_{0}$ is $1 \mathrm{~mm}$, which is twice the coarsest grid cell size.

The wave diagrams obtained on the three meshes are shown in Fig. 10. The propagation of the shocks is still unaffected by the resolution, but now the location of the mixing layer boundaries is also very close. Figure 11 illustrates that the mixing layer thickness follows a similar evolution on all the grids. Nevertheless, grid convergence does not seem to be perfectly reached with the coarsest mesh, as the behaviour of the mixing layer slightly differs from the two other cases. These observations are confirmed by the evolution of the turbulent kinetic energy, presented in Fig. 12. For a given wavelength content of the geometric perturbations and a given initial amplitude, the mixing layer thickness evolution is thus grid independent.

Because of their initial amplitude, the perturbations saturate rapidly $(\sim \tau=0.07)$, and the initial exponential growth phase is almost not observed here. Despite the initial broad spectrum, the development of the mixing layer is dominated by a single angular mode whose wavelength grows as the interface radius increases during the strong shock wave phase. This result is consistent with what is observed on HE fireballs [29]. This mode corresponds to $n=60$ on all grids. The self-similar regime does not seem to be 


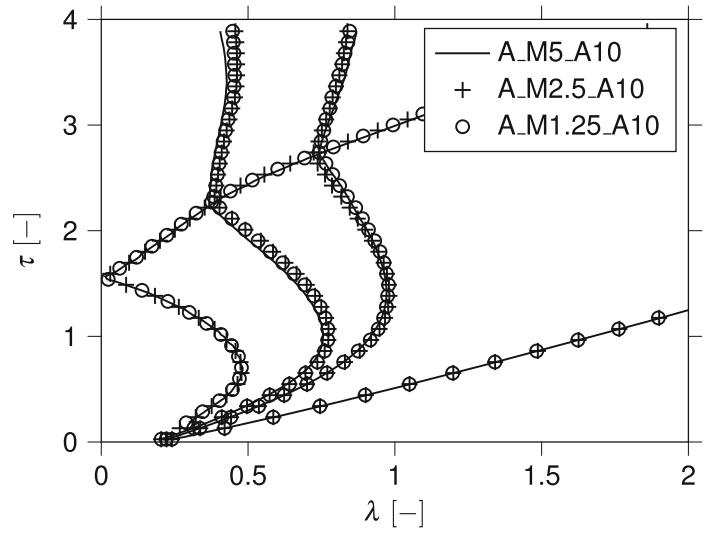

Fig. 10 Influence of the mesh resolution on the wave diagram with initial geometric perturbation of the interface

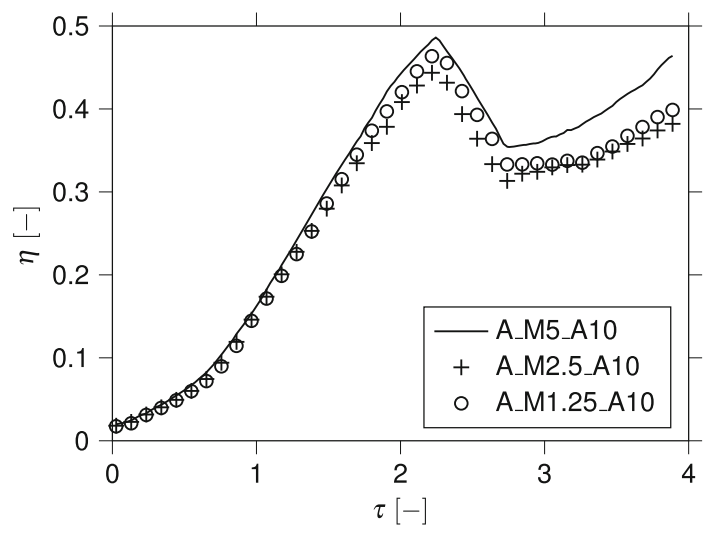

Fig. 11 Influence of the mesh resolution on the mixing layer thickness evolution with an initial geometric perturbation of the interface

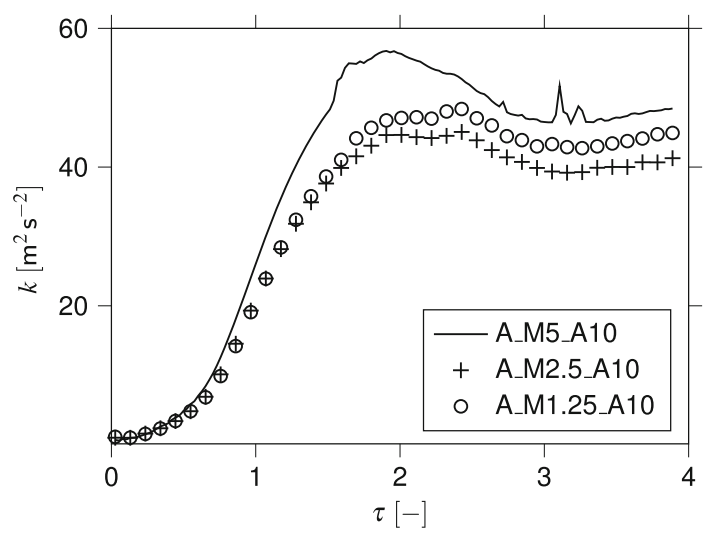

Fig. 12 Influence of the mesh resolution on the turbulent kinetic energy evolution with an initial geometric perturbation of the interface

reached until almost the end of the implosion phase as the flow clearly retains memory of the initial perturbations until then. The increase in the interface acceleration towards the centre seems to freeze the spectrum of perturbations before wavelengths greater than the currently dominant one have time to grow sufficiently.
Table 3 Summary of the experimental tests

\begin{tabular}{llll}
\hline Test label & $\varnothing(\mathrm{mm})$ & $m_{\text {glass }}(\mathrm{g})$ & $P_{1}$ (bar) \\
\hline A1 & 51 & 24.1 & 28.0 \\
A2 & & 31.5 & 28.5 \\
B1 & 73 & 53.4 & 20.0 \\
B2 & & 51.0 & 24.0 \\
\hline
\end{tabular}

\subsection{Conclusions of the 2D study}

The 2D simulations highlight the natural instability of the spherical interface. They also indicate that grid independence can be reached when taking into account initial perturbations on the interface. Their initial amplitude determines the regime of development of the instability, and their initial spectrum has a strong influence on their evolution. It should be pointed out that this grid convergence study is partial since it does not cover the whole parameter space. Moreover, it has the inherent limitations of 2D geometry so that its conclusions should be taken with care regarding generality. Nevertheless, it gives some confidence to the analysis of postdetonation turbulent mixing using numerical simulations. The phenomenology captured by the simulation is consistent with other simulations found in the literature [11,28], even the ones concerning HE fireballs. It shows that the compressed balloon analogy, which was known to be well representative of the blast-related characteristics of the flow produced by the detonation of a HE, can also be applied to study mixing inside fireballs.

\section{Experimental study}

Four tests, described in Table 3, have been conducted experimentally using the set-up described in Sect. 3. Cases A1 and A2 correspond to case A of the numerical simulations (see Table 1), and tests B1 and B2 match with case B. These tests thus allow the evaluation of the effect of the initial energy on the flow, considering a constant Atwood number, thanks to a variation of the sphere diameter and a constant initial overpressure.

From a qualitative point of view, all the distinctive features of a detonation-induced flow are visible. The schlieren records, some of which are presented in Fig. 13, show the four phases described in Sect. 1. The primary shock wave is visible in Fig. 13b as well as the external boundary of the mixing layer. The secondary shock wave can also be distinguished at the top of Fig. 13c where it appears as an arc emerging from the turbulent area.

After the sphere is shattered, glass fragments are set in motion by the flow. They can be seen in Fig. 13b, c, inside 


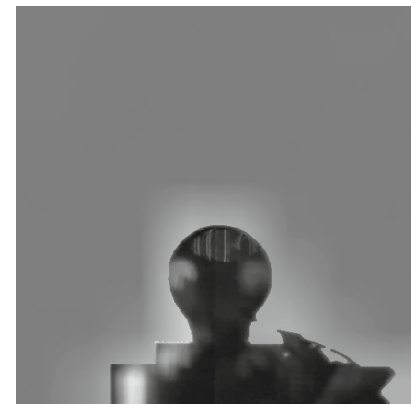

(a)

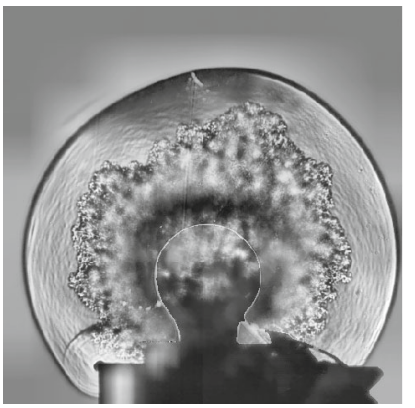

(b)

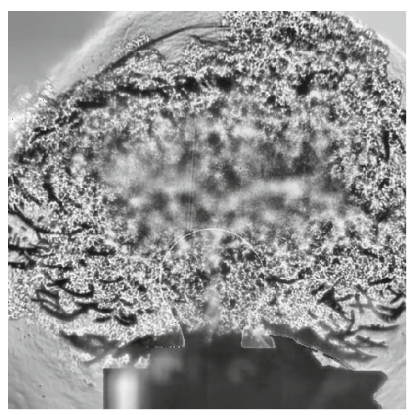

(c)

Fig. 13 Schlieren images of the experiment (test A1) after backgroundremoval and contrast enhancement. a Before explosion. b Strong blast wave phase. $\mathbf{c}$ End of reshock phase. The secondary shock is visible near the top left corner of the picture (c)

the turbulent region, as dark blurry circular zones and sharp black strips, respectively. A fraction of the initial total energy is thus converted into kinetic energy of the glass fragments and is not available to the flow. Since fragments are not taken into account in the simulations, the energy dissipated by their motion has to be subtracted from the initial energy in order to be able to compare the experimental and numerical data using the scaling law presented in Sect. 3. The evolution of the position of the fragments has been determined using the high-speed video frames and their velocity deducted from the frame rate. When possible, this value has been compared with the one measured by PDV. The agreement between the two techniques is good. Although evolving velocities are measured with both methods, the kinetic energy of the fragments is calculated using the value taken by the velocity when it reaches a plateau. This value, noted, $v_{\text {glass }}$, is given in Table 4. By knowing the total mass of glass of the sphere, it is then possible to determine the kinetic energy of the glass frag- ments. By subtracting it from the actual initial total energy of the gas, the total energy that would have been needed to achieve the same flow in the absence of glass is determined. This energy is then used for scaling with (5) and (6). The corresponding pressure calculated using $(8)$ is noted $P_{1}^{\prime}$. The corrected pressure and the scaling coefficients of each experimental test are gathered in Table 4.

It should be noted that the presence of the fragments may also affect the mixing in several ways. They indeed modify the pressure gradient across the interface and thus the baroclinic torque. Another important effect is their wake. Unfortunately, at this point, as a quantitative assessment of these effects seems out of reach, only the energy deficit due to their motion is accounted for.

Due to the residual strain in the glass caused by the metalglass sealing, the 73-mm-diameter spheres were not as strong as expected. Thus, the pressure $P_{1}^{\prime}$ reached experimentally during tests $\mathrm{B} 1$ and $\mathrm{B} 2$ is slightly lower than the one planned for case B (i.e., 22.5 bar). It is to be noted that the low velocity of the fragments obtained during test $\mathrm{A} 2$ is due to their size. The sphere indeed shattered differently than in the other tests and produced larger debris.

Figure 14 presents the wave diagrams of the four present experiments together with the data of Boyer et al. [7]. The agreement is good, especially regarding the evolution of the primary shock wave, which is the easiest to track. Similar to the experimental data of Boyer et al., the regression of the external border of the mixing layer during the implosion phase is hardly visible. However, schlieren records in the present study show that the turbulent structures located close to the centre tend to be driven towards it during that phase. The secondary shock wave location, as well as the interface external radius, shows more discrepancy, which can be attributed to several factors. First, the properties of the glass, such as its thickness or its resistance to fracturing, have an influence on the fragments behaviour and thus on the flow. The spheres used here were, for example, thicker and heavier than the one used by Boyer, which weighed between 15 and $18 \mathrm{~g}$. Then, Boyer et al. plotted their wave diagram using pictures obtained with a traditional streak camera with a rotating drum, which offered a better time and spatial resolution than the high-speed digital camera used in this work. Finally, since the spheres shattered during pressurisation, the gas inlet was still opened when the spheres broke. Thus, a jet of air was
Table 4 Corrected pressure and scaling coefficients

\begin{tabular}{llllllll}
\hline Test & $P_{0}(\mathrm{mbar})$ & $T_{0}(\mathrm{~K})$ & $v_{\text {glass }}\left(\mathrm{m} \mathrm{s}^{-1}\right)$ & $P_{1}^{\prime}(\mathrm{bar})$ & $E_{1}^{\prime}(\mathrm{J})$ & $s_{r}\left(\mathrm{~m}^{-1}\right)$ & $s_{t}\left(\mathrm{~s}^{-1}\right)$ \\
\hline A1 & 971 & 283 & 88 & 22.6 & 392 & 14.6 & 4922 \\
A2 & & 286 & 59 & 25.4 & 440 & 14.0 & 4760 \\
B1 & 970 & 276 & 80 & 16.7 & 845 & 11.3 & 3755 \\
B2 & & 277 & 80 & 20.8 & 1059 & 10.5 & 3497 \\
\hline
\end{tabular}




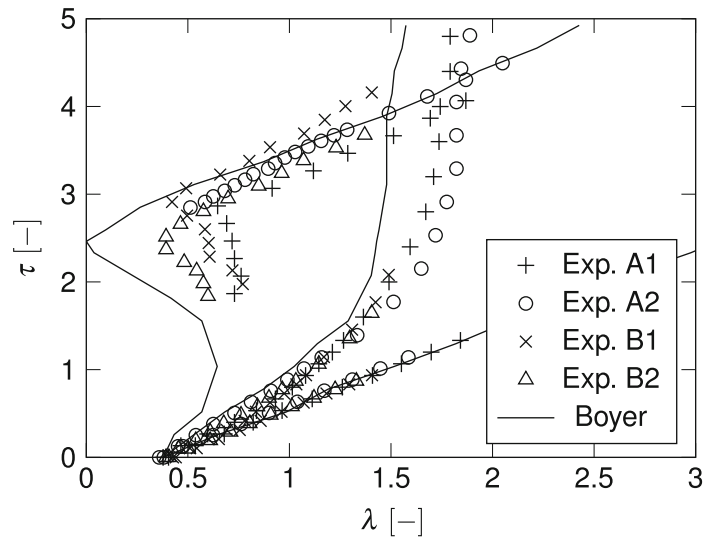

Fig. 14 Comparison of experimental wave diagrams with data from Boyer [7]. From left to right on each data set: secondary shock wave, interface external radius and primary shock wave

pushing upwards along the centre of symmetry of the flow, which explains why the secondary shock wave reflections do not occur at the origin.

Nevertheless, the four experimental tests show very similar results despite the variation of initial conditions. In particular, the variation of the sphere diameter between tests $\mathrm{A} 1, \mathrm{~A} 2$ on the one hand, and $\mathrm{B} 1, \mathrm{~B} 2$ on the other hand, does not seem to affect the dynamics of the mixing layer. Consequently, the mixing process seems to scale with the initial energy. This important conclusion is verified in the $3 \mathrm{D}$ numerical study presented in the next section.

\section{3D numerical study}

The 2D simulations conducted in Sect. 4 have shown that grid independence can be achieved when the initial perturbation of the interface is accounted for. This conclusion will now be checked in 3D. The influence of the initial energy is investigated by comparing simulations A and B. Results are then compared to the experimental data from the present study that were presented in Sect. 5. The effect of the initial density ratio is finally studied by comparing results of simulations of cases B and C. Simulations will be named using the same rationale as in the $2 \mathrm{D}$ study.

\subsection{Phenomenology}

The 3D simulations, similar to the 2D simulations, reproduce all the features of the flow as shown in Fig. 15 which shows the density gradient in a $2 \mathrm{D}$ cut of the flow obtained with simulation A_M10_A0. In Fig. 15a all the hydrodynamic phenomena occurring inside fireballs are visible. From the sphere centre, located in the bottom left corner, one first goes through the expansion wave, which appears as a gradation

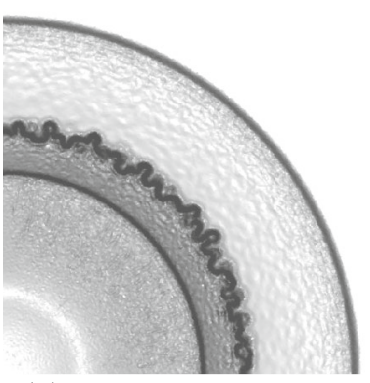

(a) Strong blast wave phase

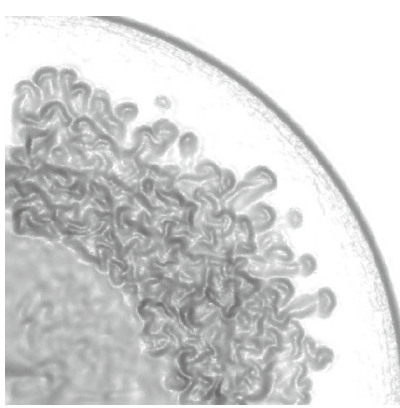

(c) End of the reshock phase

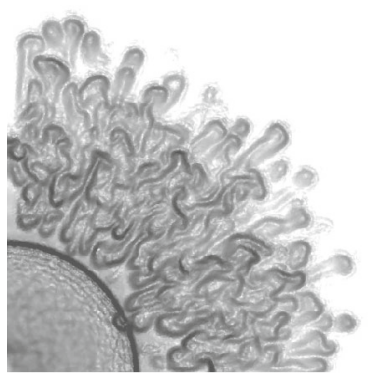

(b) End of the implosion phase

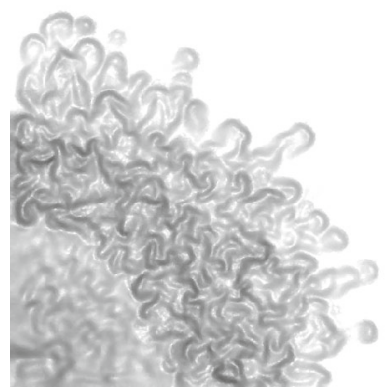

(d) Asymptotic mixing phase
Fig. 15 Density gradient in logarithmic scale obtained with the 3D simulation A_M05_A0 showing the characteristic steps of the flow. Zoom on the central area

of grey, then the secondary shock wave, the interface and finally the primary shock wave. This figure also shows that the interface is naturally unstable, as expected from theory.

Figure 16 shows a 3D view of the external boundary of the mixing layer for case A_M10_A0 with the same time sequence as Fig. 15. It highlights the fact that the turbulent structures created by RT instabilities have similar shapes and sizes and are evenly distributed on the interface until the end of the implosion phase. The most unstable mode corresponds to the lowest wavelength resolved on the grid, which is $n \approx 40$ on the grid used here for which $\Delta=1 \mathrm{~mm}$. Despite being non-reacting, these simulations are qualitatively very similar to those of the afterburning of a HE performed by Balakrishnan [13] or Kuhl [28], which confirms once more the validity of the compressed balloon method to study mixing inside HE fireballs.

\subsection{Influence of initial perturbations and grid convergence}

As in the 2D study, a composite sinusoidal perturbation has been added to the initial shape of the interface to study the influence of the mesh on the development of the mixing layer. 


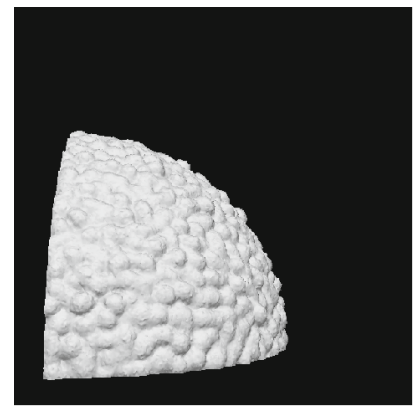

(a) Strong blast wave phase

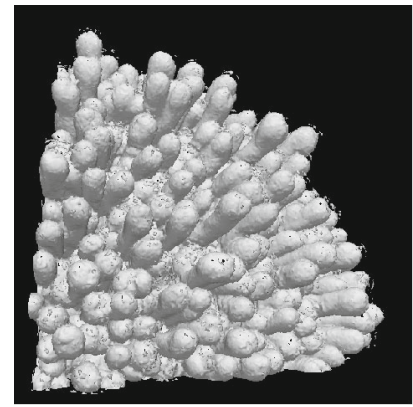

(c) End of the reshock phase

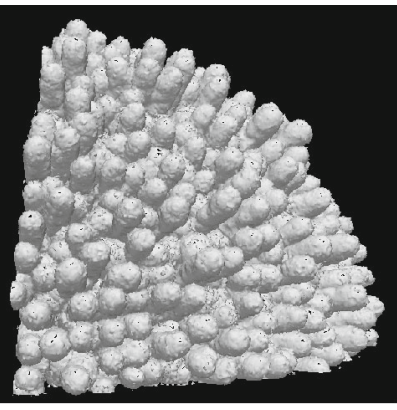

(b) End of the implosion phase

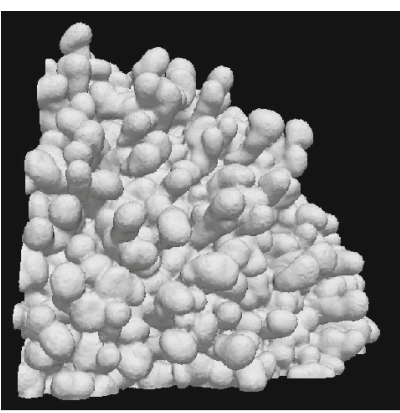

(d) Asymptotic mixing phase

Fig. $163 \mathrm{D}$ view of the external boundary of the mixing layer (isosurface $Y_{\mathrm{O}_{2}}=0.9 Y_{\mathrm{O}_{2}, 0}$ ) obtained with simulation A_M10_A0

The initial radius of the interface is:

$$
\begin{aligned}
r_{\text {int }}= & r_{\text {int }, 0} \\
& +\frac{h_{0}}{2} \frac{\sum_{n}\left[\cos \left(n \theta+\phi_{\theta, n}\right)+\cos \left(n \varphi+\phi_{\varphi, n}\right)\right]}{\left.\max \left(\sum_{n} \cos \left(n \theta+\phi_{\theta, n}\right)+\cos \left(n \varphi+\phi_{\varphi, n}\right)\right]\right)}
\end{aligned}
$$

where $\theta$ and $\varphi$ are the azimuthal and polar angle, $n$ is the mode number, and $\phi_{\theta, n}$ and $\phi_{\varphi, n}$ are random phases associated with mode $n$ and applied on the $\theta$ and $\varphi$ coordinates, respectively.

Two meshes have been used with a resolution of 1.0 and $0.5 \mathrm{~mm}$ in the central area, corresponding to 32 and 255 million cells, respectively. The same constraints on the perturbations wavelength and amplitude as in the 2D study were applied here. The mode wavelengths chosen here correspond to values of $n$ going from 20 to 40, i.e., wavelengths between 8 and $4 \mathrm{~mm}$, which is the smallest wavelength that can be resolved on the coarsest mesh. The initial amplitude of the perturbations is $h_{0}=2 \mathrm{~mm}$. The resulting wave diagrams are plotted in Fig. 17.

The agreement between the two diagrams is good despite the larger external radius of the mixing layer on the finest grid at the beginning of the implosion phase. However, it should be kept in mind that, for a reason of computational cost, the finest mesh used here has the same resolution as the coarsest grid used in the 2D study. It shows that some level of grid independence can also be achieved in 3D when initial

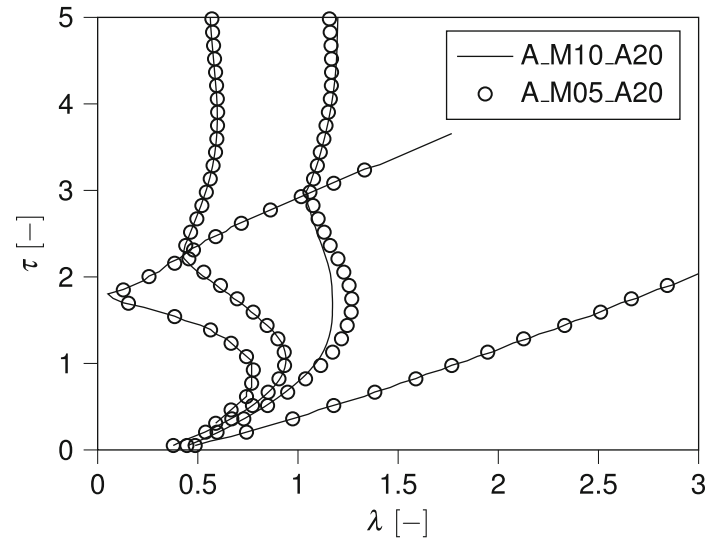

Fig. 17 Influence of the mesh resolution on the wave diagram with initial geometric perturbation of the interface for 3D simulations

perturbations are accounted for. The rest of the 3D study will then be performed on the coarsest grid.

\subsection{Comparison with the experimental results}

The wave diagram obtained with simulation A_M10_A20 is compared with the ones from the experiments in Fig. 18 which shows the temporal evolution of the positions of the primary and secondary shock waves and the position of the external boundary of the mixing layer. The numerical results show a qualitative agreement on the behaviour with the experiment and show the same features. The mixing layer radius is correctly predicted until the implosion phase, and the secondary shock wave velocity after its reflection at the centre is correctly captured. However, there is a mismatch between numerical and experimental data. The difference is attributed to the presence of the glass fragments which heavily modify the flow by confining it during the first moments after the sphere shattering and which are not taken into account in the simulations. The shock waves thus follow a slower formation process in the experiments than in the simulation, which explains the gap between experimental and numerical shock waves. The same observations were made by Boyer et al. [7] when comparing their experimental data with 1D Lagrangian simulations made by Brode [30]. Besides, the differences cannot be attributed to the scaling method since the two data sets coincide for $\tau=0$.

Figure 19 shows a comparison of a schlieren image from test A1 with an image of the gradient of the density field from simulation A_M05_A20 plotted in logarithmic scale. The image from the simulation is superimposed on the experimental picture. The scale of both pictures is approximately the same, and they correspond to a scaled time $\tau \approx 1$. The numerical and the experimental images show a good qualitative agreement. The primary shock is faster in the simulation, which is consistent with the wave diagrams (Fig. 18). The 


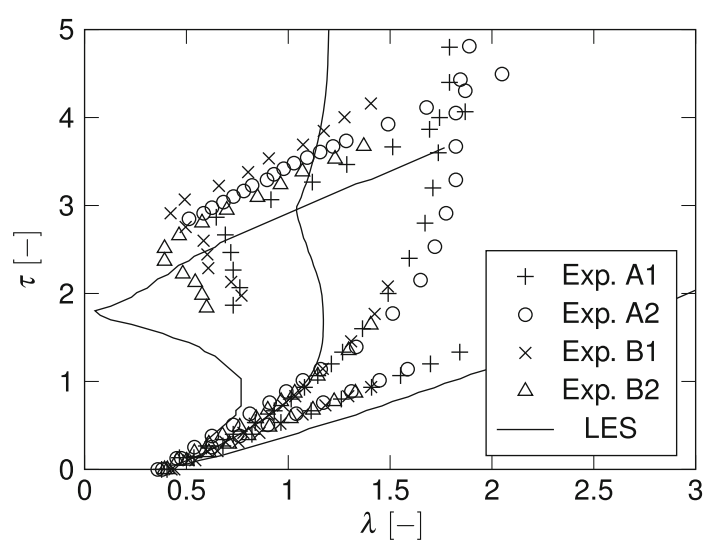

Fig. 18 Comparison of the numerical wave diagram from simulation A_M10_A20 (solid line) with experimental data (symbols). From left to right on each data set: secondary shock wave, interface external radius, and primary shock wave

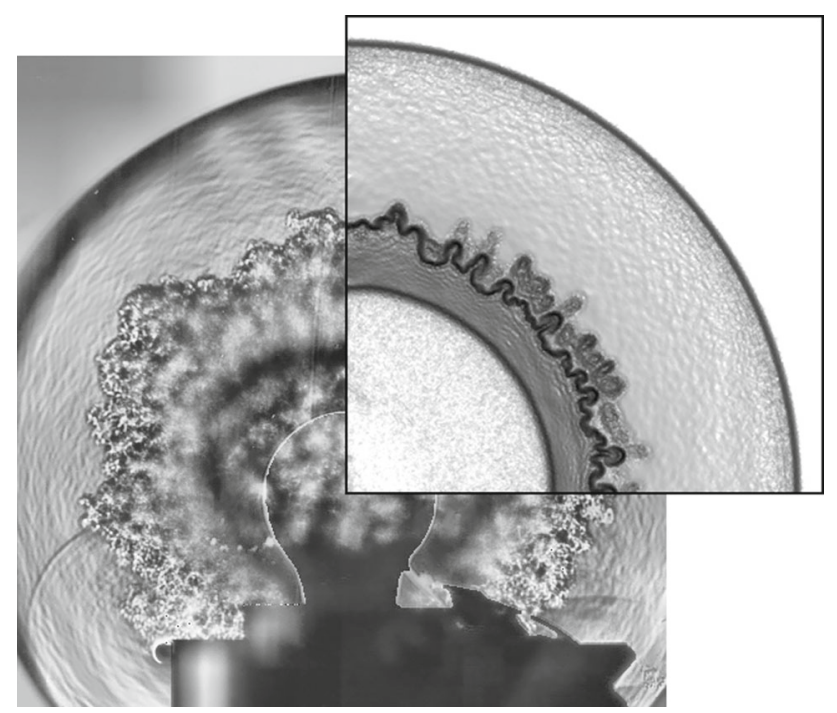

Fig. 19 Comparison of a schlieren picture from test A1 with a cut of the density gradient field from simulation A_M05_A20 at time $\tau \approx 1$

mixing layer has the same external radius and shows qualitatively similar structures on both images. Even though most of the physics is accounted for in the simulation, it should be kept in mind that the presence of the glass fragments is only considered as a correction of the initial energy of the gaseous phase. It should also be noted that the experimental image represents the density gradient field integrated along the line of sight, whereas the numerical picture is a $2 \mathrm{D}$ cut across the $3 \mathrm{D}$ field. The shape of the vortical structures created by the RT instability is thus sharper.

\subsection{Influence of the initial energy}

The influence of the initial energy on the flow is assessed by comparing simulations of cases $\mathrm{A}$ and $\mathrm{B}$ for which only the sphere diameter differs, the thermodynamic con-

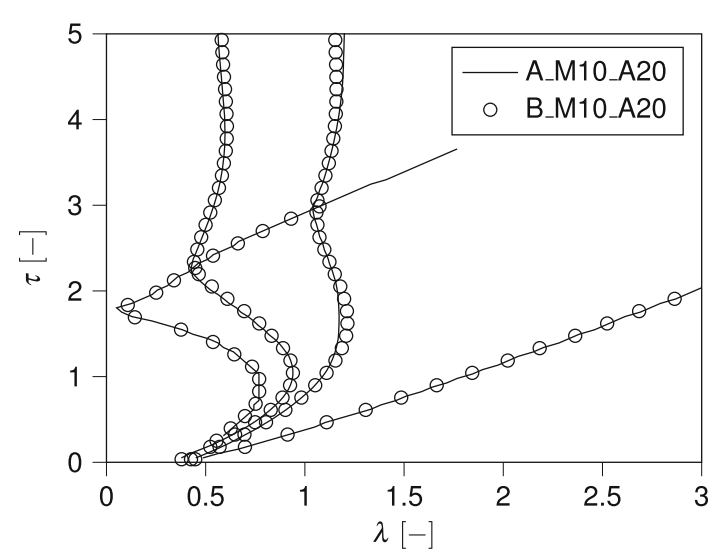

Fig. 20 Comparison of the wave diagrams obtained with the two different radii

ditions remaining unchanged. The wave diagrams, plotted in Fig. 20, show that, considering a constant Atwood number ( $A=0.91)$, the mixing process scales according to the initial total energy. As recalled in Sect. 5, this phenomenon is also observed on the experimental data since the mixing layer external radius is the same for all the four tests (Fig. 18) for which the Atwood number is almost constant since it varies between 0.91 and 0.93 .

Applied to HE, an important conclusion of this observation is that, considering a given $\mathrm{HE}$, the potential change in the mixing process caused by variation of mass is not due to the induced variation of initial energy, but is rather a consequence of the variation of the density ratio between air and detonation products at the end of the detonation, as will be shown in the next section. This result is consistent with the theory describing RT instability. The evolution of RT structures indeed depends on three parameters: the Atwood number, the perturbations wavelength and the acceleration of the interface. The first two are unchanged between the two cases, and the last one scales with the initial total energy.

The evolution of the mixing layer thickness $\eta$, presented in Fig. 21, confirms the observations made on the wave diagram. Only a slight difference is visible for late times during the asymptotic mixing phase.

\subsection{Influence of the initial density ratio}

The influence of the initial density ratio has been investigated by comparing cases $\mathrm{A}$ and $\mathrm{C}$ for which only the initial pressure differs, all other parameters remaining unchanged. The comparison of the wave diagrams obtained with simulations A_M10_A20 and C_M10_A20 is presented in Fig. 22. This time, the mixing layer is clearly affected by the change in the initial condition. It is wider and penetrates deeper towards the centre when the initial density ratio is higher.

The evolution of the mixing layer thickness is plotted in Fig. 23. It does not seem to be affected by the pressure 


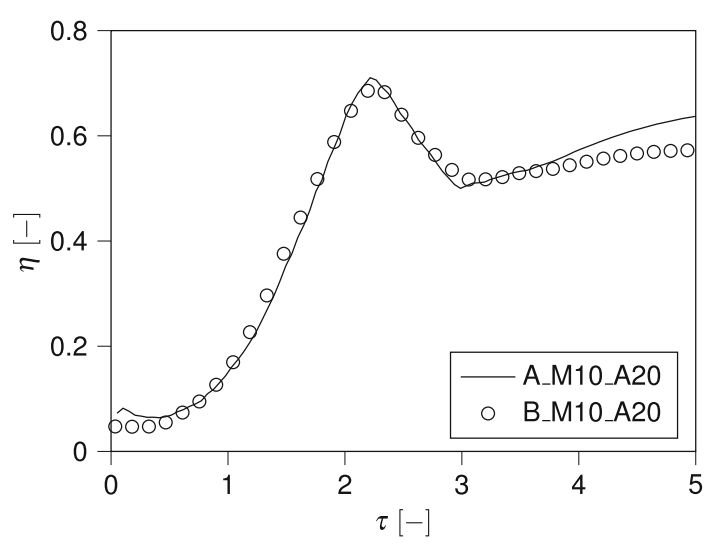

Fig. 21 Influence of the initial internal energy on the mixing layer thickness

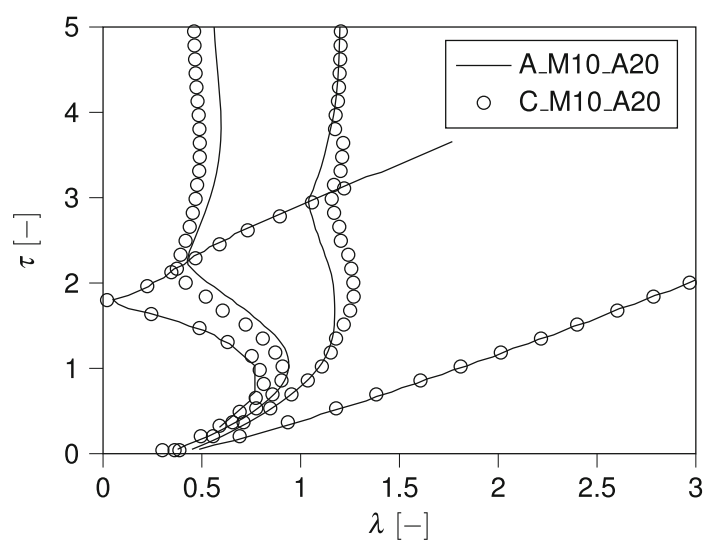

Fig. 22 Comparison of the wave diagrams obtained with the two different internal pressures

ratio during the strong blast wave phase but then grows faster during the implosion phase when the initial density ratio (or initial Atwood number) is higher. After reshock, the gap remains roughly constant. As a consequence, for an unconfined explosion, the initial density ratio between HE detonation products and air is, along with the wavelength of perturbations, one of the key parameters governing the mixing process inside fireballs.

\section{Conclusions}

The compressed balloon method is usually used to determine the blast produced by a HE charge without having to deal with the complexity related to the detonation products equation of state [8]. In this study, the same method has been used, both experimentally and numerically, to study the mixing process inside HE fireballs. This approach has proven successful in reproducing the distinctive features of such flows and yields results consistent with the few numerical simulations of HE fireballs available in the literature $[6,13,28]$. Furthermore, it

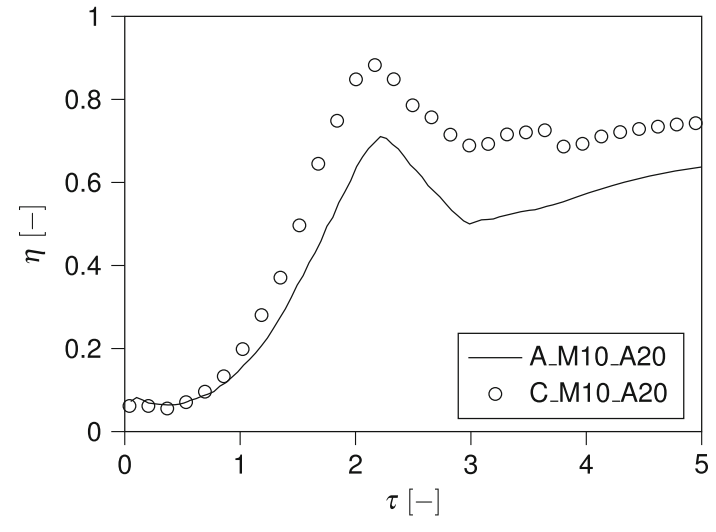

Fig. 23 Mixing layer thickness comparison between cases A and C

has the advantage of allowing the initial energy to be decoupled from the initial density ratio, which is not possible when using HE.

Numerical simulations were carried out in 2D and 3D and compared with a fair agreement to the experiments. The simulations first highlight the importance of the effect of initial perturbations on the flow. In particular, their wavelength affects the growth of the mixing layer, and their initial amplitude determines the growth regime of the RT instability. It implies that the initial perturbations have to be known in order to predict the mixing inside a fireball. Then, the mixing process seems to scale with the initial energy but rather depends on the initial density ratio. These observations are consistent with the theory of RT instability, whose development mostly depends on three factors: the perturbations wavelength, the interface acceleration, and the Atwood number.

The influence of the perturbations wavelength and amplitude has now to be investigated in greater detail. Reacting cases, for which the spheres are filled with the hot combustion products of a rich propane/air mixture, will be studied next, both experimentally and numerically, in order to focus on the combustion process.

\section{References}

1. Kuhl, A.L.: Spherical mixing layers in explosions. In: Dynamics of Exothermicity: In Honor of Antoni Kazimierz Oppenheim, pp. 291-320. Gordon and Breach (1996)

2. Rayleigh, L.: Investigation of the character of the equilibrium of an incompressible heavy fluid of variable density. Proc. Lond. Math. Soc. s1-14(1), 170-177 (1882). https://doi.org/10.1112/plms/s114.1.170

3. Taylor, G.: The instability of liquid surfaces when accelerated in a direction perpendicular to their planes. Proc. R. Soc. Lond. Ser. A 201, 192 (1950). https://doi.org/10.1098/rspa.1950.0052

4. Richtmyer, R.: Taylor instability in shock acceleration of compressible fluids. Commun. Pure Appl. Math. 13(2), 297 (1960). https:// doi.org/10.1002/cpa.3160130207 
5. Meshkov, E.E.: Instability of the interface of two gases accelerated by a shock wave. Fluid Dyn. 4(5), 101-104 (1969). https://doi.org/ 10.1007/BF01015969

6. Milne, A.M., Cargill, S.B., Longbottom, A.W.: Modelling of complex blast. Int. J. Prot. Struct. 7(3), 325-339 (2016). https://doi. org/10.1177/2041419616661431

7. Boyer, D.W.: An experimental study of the explosion generated by a pressurized sphere. J. Fluid Mech. 9, 401-429 (1960). https://doi. org/10.1017/S0022112060001195

8. Larcher, M., Casadei, F.: Explosions in complex geometries-a comparison of several approaches. Int. J. Prot. Struct. 1(2), 169195 (2010). https://doi.org/10.1260/2041-4196.1.2.169

9. Anisimov, S., Zeldovich, Y.: Rayleigh-Taylor instability of boundary between detonation products and gas in spherical explosion. Pis'ma Zh. Eksp. Teor. Fiz. 3, 1081 (1977)

10. Anisimov, S.I., Zeldovich, Y.B., Inogamov, M.A., Ivanov, M.F.: The Taylor instability of contact boundary between expanding detonation products and a surrounding gas. In: Shock Waves, Explosions, and Detonations, Progress in Astronautics and Aeronautics, vol. 87, pp. 218-227. American Institute of Aeronautics and Astronautics, New York (1983). https://doi.org/10.2514/5. 9781600865602.0218.0227

11. Kuhl, A.L.: Mixing in explosions. Technical Report. UCRL-JC115690, Lawrence Livermore National Laboratory (1993)

12. Kuhl, A.L., Ferguson, R.E., Priolo, F., Chien, K.Y., Collins, J.P.: Baroclinic mixing in HE fireballs. Technical Report. UCRL-JC114982, Lawrence Livermore National Laboratory (1993)

13. Balakrishnan, K., Genin, F., Nance, D., Menon, S.: Numerical study of blast characteristics from detonation of homogeneous explosives. Shock Waves 20, 147-162 (2010). https://doi.org/10.1007/ s00193-009-0236-4

14. Plesset, M.S.: On the stability of fluid flows with spherical symmetry. J. Appl. Phys. 25, 96-98 (1954). https://doi.org/10.1063/1. 1721529

15. Youngs, D.L.: Numerical simulation of turbulent mixing by Rayleigh-Taylor instability. Physica D 12, 32-44 (1984). https:// doi.org/10.1016/0167-2789(84)90512-8

16. Anuchina, N.N., Kucherenko, Y.A., Neuvazhaev, V.E., Ogibina, V.N., Shibarshov, L.I., Yakolev, V.G.: Turbulent mixing at an accelerating interface between liquids of different density. Fluid Dyn. 13(6), 916-920 (1978). https://doi.org/10.1007/BF01050969
17. Read, K.I.: Experimental investigation of turbulent mixing by Rayleigh-Taylor instability. Physica D 12, 45-58 (1984). https:// doi.org/10.1016/0167-2789(84)90513-X

18. Bell, G.I.: Taylor instability on cylinders and spheres in the small amplitude approximation. Technical Report. Los Alamos Scientific Laboratory (1951)

19. Mikaelian, K.O.: Rayleigh-Taylor and Richtmyer-Meshkov instabilities and mixing in stratified spherical shells. Phys. Rev. A 42(6), 3400 (1990). https://doi.org/10.1103/PhysRevA.42.3400

20. Mikaelian, K.O.: Rayleigh-Taylor and Richtmyer-Meshkov instabilities and mixing in stratified cylindrical shells. Phys. Fluids 17(9), 094105 (2005). https://doi.org/10.1063/1.2046712

21. CFD Team at CERFACS. AVBP solver. https://cerfacs.fr/en/ computational-fluid-dynamics-softwares

22. Donea, J., Huerta, A.: Finite Element Method for Flow Problems. Wiley, New York (2003). https://doi.org/10.1002/0470013826

23. Colin, O.: Simulations aux grandes échelles de la combustion turbulente prémélangée dans les statoréacteurs. PhD Thesis, Toulouse, INPT (2000)

24. Nicoud, F., Baya Toda, H., Cabrit, O., Bose, S., Lee, J.: Using singular values to build a subgrid-scale model for large eddy simulations. Phys. Fluids 23, 085106 (2011). https://doi.org/10.1063/ 1.3623274

25. von Neumann, J., Richtmyer, R.: A method for the numerical calculation of hydrodynamic shocks. J. Appl. Phys. 21, 232 (1950). https://doi.org/10.1063/1.1699639

26. Sachs, R.G.: The dependence of blast on ambient pressure and temperature. Technical Report. BRL Report 466, Aberdeen Proving Ground (1944)

27. Bach, G.G., Lee, J.H.S.: An analytical solution for blast waves. AIAA J. 8(2), 271-275 (1970). https://doi.org/10.2514/3.5655

28. Kuhl, A.L., Bell, J.B., Beckner, V.E., Balakrishnan, K., Aspden, A.J.: Spherical combustion clouds in explosions. Shock Waves 23, 233-249 (2013). https://doi.org/10.1007/s00193-012-0410-y

29. Frost, D.L., Zarei, Z., Zhang, F.: Instability of combustion products interface from detonation of heterogeneous explosives. In: 20th International Colloquium on the Dynamics of Explosions and Reactive Systems, Montreal, Canada, Paper 198 (2005)

30. Brode, H.: Blast wave from a spherical charge. Phys. Fluids 2 , 217-229 (1959). https://doi.org/10.1063/1.1705911 